\title{
Pacific
}

Journal of

Mathematics

AN APPLICATION OF HOMOGENIZATION THEORY TO HARMONIC ANALYSIS ON SOLVABLE LIE GROUPS OF POLYNOMIAL GROWTH

G. Alexopoulos

Volume $159 \quad$ No. 1

May 1993 


\section{AN APPLICATION OF HOMOGENIZATION THEORY TO HARMONIC ANALYSIS ON SOLVABLE LIE GROUPS OF POLYNOMIAL GROWTH}

\section{G. Alexopoulos}

Let $Q$ be a connected solvable Lie group of polynomial growth. Let also $E_{1}, \ldots, E_{p}$ be left invariant vector fields on $G$ that satisfy Hörmander's condition and denote by $L=-\left(E_{1}^{2}+\cdots+E_{p}^{2}\right)$ the associated sub-Laplacian and by $S(x, t)$ the ball which is centered at $x \in Q$ and it is of radius $t>0$ with respect to the control distance associated to those vector fields. The goal of this article is to prove the following Harnack inequality: there is a constant $c>0$ such that $\left|E_{i} u(x)\right| \leq c t^{-1} u(x), x \in Q, t \geq 1,1 \leq i \leq p$, for all $u \geq 0$ such that $L u=0$ in $S(x, t)$. This inequality is proved by adapting some ideas from the theory of homogenization.

0. Introduction. Let $Q$ be a connected solvable Lie group which we assume to be of polynomial growth; i.e., if $d g$ is a left invariant Haar measure on $Q$ and $V$ a compact neighborhood of the identity element $e$ of $Q$, then there are constants $c, d>0$ such that

$$
d g-\operatorname{measure}\left(V^{n}\right) \leq c n^{d}, \quad n \in \mathbb{N} .
$$

Notice that the connected nilpotent Lie groups are also solvable and of polynomial growth (cf. [5], [6]).

Let us identify the Lie algebra $\mathfrak{q}$ of $Q$ with the left invariant vector fields on $Q$ and consider $E_{1}, \ldots, E_{p} \in \mathfrak{q}$ that satisfy Hörmander's condition; i.e., together with their successive Lie brackets $\left[E_{i_{1}},\left[E_{i_{2}}\right.\right.$, $\left.\left.\left[\ldots\left[E_{i_{s-1}}, E_{i_{s}}\right] \ldots\right]\right]\right]$, they generate $\mathfrak{q}$. To these vector fields there is associated, in a canonical way, a left invariant distance $d_{E}(\cdot, \cdot)$ on $G$, called control distance. This distance has the property that (cf. [15]) if $S_{E}(x, t)=\left\{y \in G, d_{E}(x, y)<t\right\}, x \in G, t>0$ then there is $c \in \mathbb{N}$ such that

$$
S_{E}(e, n) \subseteq V^{c n}, \quad V^{n} \subseteq S_{E}(e, c n), \quad n \in \mathbb{N} .
$$

According to a classical theorem of L. Hörmander [7] the operator

$$
L=-\left(E_{1}^{2}+\cdots+E_{p}^{2}\right)
$$

is hypoelliptic.

The goal of this paper is to prove the following result: 
TheOREM 1. Let $Q, E_{1}, \ldots, E_{p}$ and $L$ be as above. Then there is $c>0$ such that

$$
\left|E_{i} u(x)\right| \leq c t^{-1} u(x), \quad x \in Q, t \geq 1,
$$

for all $u \geq 0$ such that $L u=0$ in $S_{E}(x, t), 1 \leq i \leq p$.

This is a result of technical nature, but a very useful one, when one tries to generalise the "real variable theory" to $Q$ (cf. [9], [10]). For instance, it can be used to obtain estimates for the Poisson kernel and the Green function. Another immediate consequence of Theorem 1 is that every positive harmonic function in $Q$ (i.e. every $u \geq 0$, $u \in C^{\infty}(Q)$ such that $L u=0$ in $Q$ ) is constant (cf. [13]).

When $Q$ is also nilpotent then Theorem 1 is a particular case of a more general result of $N$. Th. Varopoulos [14], namely for all integers $l \geq 0$ there is $c_{l}>0$ such that

$$
\left|E_{i_{1}} \cdots E_{i_{l}} u(x)\right| \leq c_{l} t^{-l} u(x), \quad x \in Q, t \geq 1,
$$

for all $u \geq 0$ such that $L u=0$, in $S_{E}(x, t)$.

As we shall see, for $l \geq 2,(0.3)$ is not true for general, not necessarily nilpotent, solvable Lie groups.

(0.3) is also true for $0<t<1$ (cf. N. Th. Varopoulos [14]), but this is a local result and the Lie group structure does not play any role in proving it.

The main contribution of this article is the observation that the operator $L$ can be viewed as a second order differential operator with quasiperiodic coefficients on the nil-shadow $Q_{N}$ of $Q$, which is a nilpotent Lie group (cf. [6]). Once we adopt this point of view, proving Theorem 1 becomes a matter of generalizing results, already known for second order uniformly elliptic differential operators with periodic coefficients (cf. [1], [2]). Indeed, in that context, Theorem 1 has already been proved by $M$. Avellaneda and F. H. Lin [1].

More precisely, let (we use the summation convention for repeated indices)

$$
L_{1}=-\frac{\partial}{\partial x_{i}} a_{i j}(x) \frac{\partial}{\partial x_{j}}
$$

be a uniformly elliptic operator in $\mathbb{R}^{n}$ and assume that its coefficients $a_{i j}(x)$ are periodic (i.e. $a_{i j}(x+z)=a_{i j}(x), x \in \mathbb{R}^{n}, z \in \mathbb{Z}^{n}$ ) and Hölder continuous (i.e. there is $\alpha \in(0,1)$ and $M>0$ such that $\left.\left\|a_{i j}(x)\right\|_{C^{\alpha}\left(\mathbb{R}^{n}\right)} \leq M\right)$.

Also let

$$
L_{\varepsilon}=-\frac{\partial}{\partial x_{i}} a_{i j}\left(\frac{x}{\varepsilon}\right) \frac{\partial}{\partial x_{j}}, \quad 0<\varepsilon \leq 1,
$$


and denote by $B(x, t)$ the. Euclidean ball of radius $t>0$ centered at $x \in \mathbb{R}^{n}$.

We observe that $L_{\varepsilon} u_{\varepsilon}=0$ in $B(0,1)$ if and only if $L u=0$ in $B(0, t)$, where $u(x)=u_{\varepsilon}(\varepsilon x), t=\varepsilon^{-1}$. Hence, proving that there is $c>0$ such that

$$
|\nabla u(0)| \leq c t^{-1} u(0), \quad t \geq 1,
$$

for all $u \geq 0$ such that $L u=0$ in $B(0, t)$, is equivalent to proving that there is a constant $c>0$, independent of $\varepsilon \in(0,1)$ such that

$$
\left|\nabla u_{\varepsilon}(0)\right| \leq c u_{\varepsilon}(0), \quad 0<\varepsilon \leq 1,
$$

for all $u_{\varepsilon} \geq 0$ such that $L_{\varepsilon} u_{\varepsilon}=0$ in $B(0,1)$. This follows from the following result of M. Avellaneda and F. H. Lin [1], using Moser's Harnack inequality (cf. [9]).

THEOREM 0.1 (cf. M. Avellaneda and F. H. Lin [1]). Let $L_{\varepsilon}, 0<\varepsilon \leq$ 1 , be as above, $f \in L^{n+\delta}(B(0,1)), \delta>0$, and $g \in C^{1, \nu}(\partial B(0,1))$, $0<\nu \leq 1$. Then there is a constant $c>0$ depending only on $\alpha, M, n, \nu, \delta$ and independent of $\varepsilon$ such that

$$
\begin{aligned}
& {\left[u_{\varepsilon}\right]_{C^{0,1}(B(0,1))}} \\
& \quad \leq c\left([g]_{C^{1, \nu}(\partial B(0,1))}+\|f\|_{L^{n+\delta}(B(0,1))}\right), \quad 0<\varepsilon \leq 1,
\end{aligned}
$$

for all $u_{\varepsilon}$ satisfying

$$
L_{\varepsilon} u_{\varepsilon}=f \text { in } B(0,1), \quad u_{\varepsilon}=g \text { on } \partial B(0,1), \quad 0<\varepsilon \leq 1 \text {. }
$$

Notice that although we do not have any, uniform with respect to $\varepsilon$, control of the Hölder continuity of the coefficients of the operators $L_{\varepsilon}$, the above result gives a uniform with respect to the $\varepsilon$ estimate for $\left[u_{\varepsilon}\right]_{C^{0,1}}$. This is due to the fact that there is an elliptic operator with constant coefficients

$$
L_{0}=-\frac{\partial}{\partial x_{i}} q_{i j} \frac{\partial}{\partial x_{j}}
$$

called the homogenized operator, which has the property that if

$$
L_{0} u_{0}=f \text { in } B(0,1), \quad u_{0}=g \text { on } \partial B(0,1), \quad 0<\varepsilon \leq 1,
$$

then

$$
u_{\varepsilon} \rightarrow u_{0}, \quad \varepsilon \rightarrow 0
$$

uniformly on the compact subsets of $B(0,1)$. 
The coefficients $q_{i j}$ of the homogenized operator $L_{0}$ are given by the formula

$$
q_{i j}=\int_{D}\left[a_{i j}(x)-a_{i l}(x) \frac{\partial}{\partial x_{l}} \chi^{j}(x)\right] d x, \quad D=[0,1]^{n}
$$

where the functions $\chi^{j}, j=1, \ldots, n$, called correctors, are the unique solutions of the problem

$$
\begin{gathered}
L\left(x_{j}-\chi^{j}\right)=0, \quad \chi^{j}(x+z)=\chi^{j}(x), \quad x \in \mathbb{R}^{n}, \quad z \in \mathbb{Z}^{n}, \\
\int_{D} \chi^{j}(x) d x=0, \quad D=[0,1]^{n} .
\end{gathered}
$$

The motivating example is the universal covering $G$ of the group of Euclidean motions on the plane, which is a three dimensional solvable Lie group of polynomial growth. It turns out that every operator $L$, as in Theorem 1 , in $G$, can be expressed as a second order differential operator in $\mathbb{R}^{3}$ with periodic coefficients.

More precisely, let $\mathfrak{g}$ denote the Lie algebra of $G$ and identify its elements with the left invariant vector fields on $G$. Then, there is a basis $\left\{X_{1}, X_{2}, X_{3}\right\}$ of $\mathfrak{g}$ such that

$$
\left[X_{1}, X_{2}\right]=X_{3}, \quad\left[X_{1}, X_{3}\right]=-X_{2}, \quad\left[X_{2}, X_{3}\right]=0 .
$$

Identifying the simply connected analytic subgroups of $G$ whose Lie algebras are generated by $\left\{X_{2}, X_{3}\right\}$ and $\left\{X_{1}\right\}$ with $\mathbb{R}^{2}$ and $\mathbb{R}$ respectively, we can see that $G$ is isomorphic to the semidirect product $\mathbb{R}^{2} \times_{\tau} \mathbb{R}$ where the action $\tau$ of $\mathbb{R}$ on $\mathbb{R}^{2}$ is given by $\tau: \mathbb{R} \rightarrow$ $L\left(\mathbb{R}^{2}\right): x \rightarrow \operatorname{rot}_{x}, \operatorname{rot}_{x}$ being the counterclockwise rotation by angle $x$ and $L\left(\mathbb{R}^{2}\right)$ the space of linear transformations of $\mathbb{R}^{2}$.

Let us consider the exponential coordinates of the second kind (cf. [12])

$$
\varphi: \mathbb{R}^{3} \rightarrow G, \quad \varphi:\left(x_{3}, x_{2}, x_{1}\right) \rightarrow \exp x_{3} X_{3} \exp x_{2} X_{2} \exp x_{1} X_{1} .
$$

If $x=\left(x_{3}, x_{2}, x_{1}\right)$, then we have (cf. $\left.\S 2\right)$

$$
\begin{aligned}
& d \varphi^{-1} X_{1}(x)=\frac{\partial}{\partial x_{1}} \\
& d \varphi^{-1} X_{2}(x)=\cos x_{1} \frac{\partial}{\partial x_{2}}+\sin x_{1} \frac{\partial}{\partial x_{3}} \\
& d \varphi^{-1} X_{3}(x)=-\sin x_{1} \frac{\partial}{\partial x_{2}}+\cos x_{1} \frac{\partial}{\partial x_{3}} .
\end{aligned}
$$

Let us now use $\varphi$ to identify $Q$ and $\mathbb{R}^{3}$ as differential manifolds. 
Let

$E_{1}=X_{1}, \quad E_{2}=X_{1}+X_{2}, \quad E_{3}=X_{3} \quad$ and $\quad L=-\left(E_{1}^{2}+E_{2}^{2}+E_{3}^{2}\right)$.

Then $L$ becomes a uniformly elliptic differential operator on $\mathbb{R}^{n}$, which can be written in divergence form as

$$
L=-\frac{\partial}{\partial x_{i}} a_{i j}(x) \frac{\partial}{\partial x_{j}}
$$

with

$$
\begin{gathered}
a_{11}=2, \quad a_{22}=a_{33}=1, \quad a_{12}=a_{21}=\cos x_{1}, \\
a_{13}=a_{31}=\sin x_{1}, \quad a_{23}=a_{32}=0 .
\end{gathered}
$$

Moreover, the control distance $d_{E}(\cdot, \cdot)$ associated to the vector fields $E_{1}, E_{2}, E_{3}$ becomes equivalent to the Euclidean one; i.e., $\exists b \geq$ $a>0$ such that $a|x-y| \leq d_{E}(x, y) \leq b|x-y|, x, y \in \mathbb{R}^{3}$.

Let us now see why the inequalities (0.3) are not true for $l \geq 2$.

Let us put

$$
L_{\varepsilon}=-\frac{\partial}{\partial x_{i}} a_{i j}\left(\frac{x}{\varepsilon}\right) \frac{\partial}{\partial x_{j}}, \quad 0<\varepsilon \leq 1 .
$$

Then proving $(0.3)$ for $l \geq 2$ and $i_{1}=i_{2}=1$ is equivalent to proving that there is $c>0$, independent of $\varepsilon$, such that

$$
\left|\frac{\partial^{2}}{\partial x_{1}^{2}} u_{\varepsilon}(0)\right| \leq c u(0), \quad 0<\varepsilon \leq 1,
$$

for all $u_{\varepsilon} \geq 0$ satisfying $L_{\varepsilon} u_{\varepsilon}=0$ in $B(0,1)$.

As we are going to see, $(0.6)$ is not true.

In the example we consider, we have that

$$
\chi^{1}(x)=0, \quad \chi^{2}(x)=\frac{1}{2} \sin x_{1}, \quad \chi^{3}(x)=-\frac{1}{2} \cos x_{1}
$$

and

$$
L_{0}=-\left(2 \frac{\partial^{2}}{\partial x_{1}^{2}}+\frac{3}{4} \frac{\partial^{2}}{\partial x_{2}^{2}}+\frac{5}{4} \frac{\partial^{2}}{\partial x_{3}^{2}}\right) .
$$

Also $L_{\varepsilon}$ can be written as

$$
\begin{aligned}
L_{\varepsilon}= & -2 \frac{\partial^{2}}{\partial x_{1}^{2}}-2 \cos \frac{x_{1}}{\varepsilon} \frac{\partial^{2}}{\partial x_{1} \partial x_{2}}-2 \sin \frac{x_{1}}{\varepsilon} \frac{\partial^{2}}{\partial x_{1} \partial x_{3}} \\
& -\frac{\partial^{2}}{\partial x_{2}^{2}}-\frac{\partial^{2}}{\partial x_{3}^{2}}+\frac{1}{\varepsilon} \sin \frac{x_{1}}{\varepsilon} \frac{\partial}{\partial x_{2}}-\frac{1}{\varepsilon} \cos \frac{x_{1}}{\varepsilon} \frac{\partial}{\partial x_{3}}
\end{aligned}
$$


Let us take $f=0$ and $g=x_{3}+2$ in (0.4). Then $u_{0}=x_{3}+2$. Hence $u_{0} \geq 0, \partial u / \partial x_{3}=1$ and $\partial u_{0} / \partial x_{1}=\partial u_{0} / \partial x_{2}=0$.

Since $L_{\varepsilon} \partial u_{\varepsilon} / \partial x_{i}=\left(\partial / \partial x_{i}\right) L_{\varepsilon} u_{\varepsilon}=0, i=2,3$, it follows from Theorem 0.1 that

(0.8) $\quad u_{\varepsilon} \rightarrow u_{0} \quad$ and $\quad \frac{\partial}{\partial x_{i}} u_{\varepsilon} \rightarrow \frac{\partial}{\partial x_{i}} u_{0}, \quad(\varepsilon \rightarrow 0), i=2,3$, uniformly on the compact subsets of $B(0,1)$ and that there is $c>0$ such that (0.9) $\left|\frac{\partial}{\partial x_{i}} \frac{\partial}{\partial x_{j}} u_{\varepsilon}(x)\right| \leq c, \quad x \in B(0,1), i=1,2,3, j=2,3$. Now, (0.7), (0.8) and (0.9) imply that

$$
\frac{\partial^{2}}{\partial x_{1}^{2}} u_{\varepsilon}(0) \sim \frac{1}{\varepsilon}, \quad(\varepsilon \rightarrow 0)
$$

which disproves (0.6). So (0.3) is not true for $l \geq 0$.

Acknowledgment. I wish to thank Professor F. Murat for several helpful discussions on the theory of homogenization. I also want to thank the referee for several suggestions.

1. The structure of the Lie algebra. Let $\mathfrak{q}$ be a solvable Lie algebra and denote by $\mathfrak{n}$ its nil-radical. Then $\mathfrak{n}$ is a nilpotent ideal of $\mathfrak{q}$ and $[\mathfrak{q}, \mathfrak{q}] \subseteq \mathfrak{n}$ (cf. [12]). We denote by $\pi$ the natural map $\pi: \mathfrak{q} \rightarrow \mathfrak{q} / \mathfrak{n}$. We also put $k=\operatorname{dim}(\mathfrak{q} / \mathfrak{n})$.

Let ad $X=S(X)+K(X)$ denote the Jordan decomposition of the derivation ad $X(Y)=[X, Y], X \in \mathfrak{q} . S(X)$ is the semisimple and $K(X)$ the nilpotent part. It is well known that

(i) $S(X)$ and $K(X)$ are derivations of $\mathfrak{q}$ (cf. [12]).

(ii) There are real polynomials $s(x)$ and $k(x)$ such that

(1.1) $\quad S(X)=s(\operatorname{ad} X)$ and $K(X)=k(\operatorname{ad} X)$ (cf. [8]).

(1.2) (iii) $[S(X), K(X)]=0$.

Notice that the fact that $\operatorname{ad} X(X)=[X, X]=0, X \in \mathfrak{q}$ implies that the constant coefficients of the polynomials $k(x)$, hence also of the polynomials $s(x)$, are zero.

LEMMA 1.1. There are vectors $Y_{1}, \ldots, Y_{k} \in \mathfrak{q}$ such that

(a) $\left[S\left(Y_{i}\right), S\left(Y_{j}\right)\right]=0,1 \leq i, j \leq k$,

(b) $\left\{\pi\left(Y_{1}\right), \ldots, \pi\left(Y_{k}\right)\right\}$ is a basis of $\mathfrak{q} / \mathfrak{n}$. 
Proof. Let $\left\{Z_{1}, \ldots, Z_{k}\right\}$ any choice of vectors of $\mathfrak{q}$ such that $\left\{\pi\left(Z_{1}\right), \ldots, \pi\left(Z_{k}\right)\right\}$ is a basis of $\mathfrak{q} / \mathfrak{n}$. To prove the lemma it is enough to prove that for every integer $1 \leq m \leq k$ we can choose vectors $Y_{1}, \ldots, Y_{m} \in \mathfrak{q}$ such that

$$
\begin{gathered}
{\left[S\left(Y_{i}\right), S\left(Y_{j}\right)\right]=0, \quad 1 \leq i, j \leq m,} \\
\left\{\pi\left(Y_{1}\right), \ldots, \pi\left(Y_{m}\right), \pi\left(Z_{m+1}\right), \ldots, \pi\left(Z_{k}\right)\right\} \text { basis of } \mathfrak{q} / \mathfrak{n} .
\end{gathered}
$$

(1.3) will be proved by induction on $m$. For $m=1$ it is enough to take $Y_{1}=Z_{1}$. So assume that $(1.3)$ is true for $m=j, 1 \leq j<k$. To prove that it is also true for $m=j+1$ assume that the vectors $Y_{1}, \ldots, Y_{j}$ have been chosen and consider the linear space $\mathfrak{b}$ that is generated by $\mathfrak{n}$ and the vectors $Z_{j+1}, \ldots, Z_{k}$. It follows from the fact that $[\mathfrak{q}, \mathfrak{q}] \subseteq \mathfrak{n}$ that $\mathfrak{b}$ is actually an ideal of $\mathfrak{q}$. By our induction hypothesis the derivations $S\left(Y_{1}\right), \ldots, S\left(Y_{j}\right)$ commute. They are also semisimple linear transformations and satisfy $S\left(Y_{i}\right)(\mathfrak{b}) \subseteq \mathfrak{n}$. This last assertion follows from the fact that the polynomials $k(x)$ and $s(x)$, in (1.1), have zero constant coefficients. Hence $\mathfrak{b}$ admits a subspace $\mathfrak{d}$ complementary to $\mathfrak{n}$, i.e., such that $\mathfrak{b}=\mathfrak{d} \oplus \mathfrak{n}$ and $S\left(Y_{i}\right)(\mathfrak{d})=\{0\}$, $1 \leq i \leq j$. For $Y_{j+1}$ we choose any non zero element of $\mathfrak{d}$ such that $\pi\left(Y_{j+1}\right)$ is linearly independent of the vectors $\left\{\pi\left(Z_{j+2}\right), \ldots, \pi\left(Z_{k}\right)\right\}$. $S\left(Y_{j+1}\right)$ will commute with the $S\left(Y_{1}\right), \ldots, S\left(Y_{j}\right)$ because of $(1.1)$ and the fact that $S\left(Y_{i}\right) Y_{j+1}=0,1 \leq i \leq j$. This proves (1.3) and the lemma follows.

Proposition 1.2. There are vectors $X_{1}, \ldots, X_{k} \in \mathfrak{q}$, such that

(a) $S\left(X_{i}\right) X_{j}=0,1 \leq i, j \leq k$.

(b) $\left\{\pi\left(X_{1}\right), \ldots, \pi\left(X_{k}\right)\right\}$ is a basis of $\mathfrak{q} / \mathfrak{n}$.

Proof. Let $\left\{Y_{1}, \ldots, Y_{k}\right\}$ be a set of elements of $\mathfrak{q}$ as in Lemma 1.1. Arguing in the same way as in the proof of that lemma we can see that $\mathfrak{q}$ has a subspace $\mathfrak{b}$ complementary to $\mathfrak{n}$, i.e. such that $\mathfrak{q}=\mathfrak{n} \oplus \mathfrak{b}$ and $S\left(Y_{i}\right) \mathfrak{b}=\{0\}, 1 \leq i \leq k$.

Let $N_{1}, \ldots, N_{k} \in \mathfrak{n}$ such that $X_{i}=Y_{i}-N_{i} \in \mathfrak{b}, i=1, \ldots, k$. The vectors $X_{1}, \ldots, X_{k}$ have all the properties required by the proposition: they satisfy (b) since they form a basis of $\mathfrak{b}$. To verify that they satisfy $S\left(X_{i}\right) X_{j}=0,1 \leq i, j \leq k$ observe that if this weren't true then we would have $\left(\operatorname{ad} X_{i}\right)^{n} X_{j} \neq 0, n \in \mathbb{N}$. To see that this is not possible let us observe first that since $K\left(Y_{i}\right)$ is a derivation we have that $\left[K\left(Y_{i}\right), \operatorname{ad} N_{i}\right]=\operatorname{ad}\left(K\left(Y_{i}\right) N_{i}\right)$, which combined with the fact that $K\left(Y_{i}\right) N_{i} \in \mathfrak{n}$ implies that the linear transformation 
$\left[K\left(Y_{i}\right), \operatorname{ad} N_{i}\right]$ is nilpotent. This in turn implies that although the $K\left(Y_{i}\right)$ and ad $N_{i}$ do not commute, we can nevertheless find $m \in \mathbb{N}$ such that $\left(K\left(Y_{i}\right)+\operatorname{ad} N_{i}\right)^{m}=0$, i.e. $K\left(Y_{i}\right)+\operatorname{ad} N_{i}$ is a nilpotent transformation.

Next we observe that

$$
\operatorname{ad} X_{i}\left(X_{j}\right)=\left(\operatorname{ad} Y_{i}+\operatorname{ad} N_{i}\right) X_{j}=\left(K\left(Y_{i}\right)+\operatorname{ad} N_{i}\right) X_{i}
$$

and that

$$
\operatorname{ad} X_{i}\left(K\left(Y_{i}\right)+\operatorname{ad} N_{i}\right)^{n}=\left(K\left(Y_{i}\right)+\operatorname{ad} N_{i}\right)^{n+1}+S\left(Y_{i}\right)\left(K\left(Y_{i}\right)+\operatorname{ad} N_{i}\right)^{n} \text {. }
$$

We also have that $\left[S\left(Y_{i}\right)\right.$, ad $\left.N_{i}\right]=0$, since

$$
0=S\left(Y_{i}\right) X_{i}=S\left(Y_{i}\right)\left(Y_{i}-N_{i}\right)=-S\left(Y_{i}\right) N_{i} .
$$

So using (1.2) we can conclude that

$$
\begin{aligned}
\operatorname{ad} X_{i}\left(K\left(Y_{i}\right)+\operatorname{ad} N_{i}\right)^{n}= & \left(K\left(Y_{i}\right)+\operatorname{ad} N_{i}\right)^{n+1} \\
& +\left(K\left(Y_{i}\right)+\operatorname{ad} N_{i}\right)^{n} S\left(Y_{i}\right), \quad n \geq 0 .
\end{aligned}
$$

From this observation we can easily see that it can be proved by induction that

$$
\left(\operatorname{ad} X_{i}\right)^{n} X_{j}=\left(K\left(Y_{i}\right)+\operatorname{ad} N_{i}\right)^{n} X_{j}, \quad n \in \mathbb{N} .
$$

This contradicts the assumption that $\left(\operatorname{ad} X_{i}\right)^{n} X_{j} \neq 0, n \in \mathbb{N}$, because the transformation $K\left(Y_{i}\right)+\operatorname{ad} N_{i}$ as we have already seen is nilpotent.

In what follows we shall consider and fix, once and for all, vectors $X_{1}, \ldots, X_{k} \in \mathfrak{q}$ having the properties described in the above proposition.

The nil-shadow $\mathfrak{q}_{N}$ of $\mathfrak{q}$. We can easily see that the conditions

$$
\begin{gathered}
{\left[X_{i}, X_{j}\right]_{N}=\left[X_{i}, X_{j}\right],\left[X_{i}, Y\right]_{N}=K\left(X_{i}\right) Y,} \\
{[Y, Z]_{N}=[Y, Z], \quad 1 \leq i, j \leq k, Y, Z \in \mathfrak{n},}
\end{gathered}
$$

define a unique product $[\cdot, \cdot]_{N}$ on the linear space $\mathfrak{q}$. We can verify directly (writing the elements $X$ of $\mathfrak{q}$ as a sum $X=X^{\prime}+Y$ with $X^{\prime}$ a linear combination of the vectors $X_{1}, \ldots, X_{k}$ and $\left.Y \in \mathfrak{n}\right)$ that $[\cdot, \cdot]_{N}$ satisfies the Jacobi identity. So, $\mathfrak{q}_{N}=\left(\mathfrak{q},[\cdot, \cdot]_{N}\right)$ is a Lie algebra, which is also nilpotent. $\mathfrak{q}_{N}$ is called the nil-shadow of $\mathfrak{q}$.

The filtration of $\mathfrak{q}$. We put $\mathfrak{r}_{1}=\mathfrak{q}$ and $\mathfrak{r}_{i+1}=\left[\mathfrak{r}_{1}, \mathfrak{r}_{i}\right]_{N}, i \geq 1$. Then, since $\mathfrak{q}_{N}$ is nilpotent, we have the following filtration of $\mathfrak{q}$ :

$$
\mathfrak{q}=\mathfrak{r}_{1} \supseteq \mathfrak{n} \supseteq \mathfrak{r}_{2} \supseteq \cdots \supseteq \mathfrak{r}_{m} \supseteq \mathfrak{r}_{m+1}=\{0\}, \quad \mathfrak{r}_{m} \neq\{0\} .
$$


Proposition 1.3. (1) $\mathfrak{r}_{1} \supseteq \mathfrak{n} \supseteq \mathfrak{r}_{2}$.

(2) $\mathfrak{r}_{i}$ is an ideal of $\mathfrak{q}$, i.e. $\left[\mathfrak{q}, \mathfrak{r}_{i}\right] \subseteq \mathfrak{r}_{i}, i=1,2, \ldots$.

(3) There are subspaces $\mathfrak{a}_{1}, \ldots, \mathfrak{a}_{m}$ of $\mathfrak{q}$ such that

(a) $S\left(X_{j}\right) \mathfrak{a}_{i} \subseteq \mathfrak{a}_{i}, j=1, \ldots, k, i=1, \ldots, m$,

(b) $\mathfrak{r}_{i}=\mathfrak{a}_{i} \oplus \cdots \oplus \mathfrak{a}_{m}$ and

(c) $\mathfrak{a}_{i}=\mathfrak{a}_{0 i} \oplus \mathfrak{a}_{1 i}$, where $\mathfrak{a}_{0 i}=\left\{Y \in \mathfrak{a}_{i}, S\left(X_{j}\right) Y=0,1 \leq j \leq\right.$ $k\}, S\left(X_{j}\right) \mathfrak{a}_{1 i} \subseteq \mathfrak{a}_{1 i}, 1 \leq j \leq k$.

Proof. (1) follows from the fact that $[\mathfrak{q}, \mathfrak{q}] \subseteq \mathfrak{n}$ and the way $[\cdot, \cdot]_{N}$ was defined. (2) can be proved by induction. It is trivially true for $i=1$. So, assume that it is true for $i=n$. We are going to verify that it is also true for $i=n+1$.

Let $X \in \mathfrak{q}, Y \in \mathfrak{r}_{1}, Z \in \mathfrak{r}_{i}$. If $X \in \mathfrak{n}$, then $\operatorname{ad} X\left([Y, Z]_{N}\right)=$ $\left[X,[Y, Z]_{N}\right]_{N} \in \mathfrak{r}_{n+2} \subseteq \mathfrak{r}_{n+1}$. If $Z \in \mathfrak{n}, Y=X_{j}$ and $X=X_{l}$ for some $1 \leq j, l \leq k$, then $\operatorname{ad} X_{l}\left(\left[X_{j}, Z\right]_{N}\right)=\operatorname{ad} X_{l} K\left(X_{j}\right) Z=$ $K\left(X_{l}\right) K\left(X_{j}\right) Z+S\left(X_{l}\right) K\left(X_{j}\right) Z=K\left(X_{l}\right) K\left(X_{j}\right) Z+K\left(X_{j}\right) S\left(X_{l}\right) Z$, since $S\left(X_{l}\right) X_{j}=0, S\left(X_{l}\right)$ is a derivation and $K\left(X_{j}\right)$ is a polynomial in ad $X_{j}$. Hence ad $X_{l}\left(\left[X_{j}, Z\right]_{N}\right)=\left[X_{l},\left[X_{j}, Z\right]_{N}\right]_{N}+\left[X_{j}, S\left(X_{l}\right) Z\right]_{N} \in$ $\mathfrak{r}_{n+1}$. Finally, if $X=X_{h}, Y=X_{l}$ and $Z=X_{j}$ for some $1 \leq$ $h, l, j \leq k$, then ad $X_{h}\left(\left[X_{l}, X_{j}\right]_{N}\right)=\left[X_{h},\left[X_{l}, X_{j}\right]_{N}\right]_{N} \in \mathfrak{r}_{n+2} \subseteq$ $\mathfrak{r}_{n+1}$. Since the general case is a linear combination of the cases examined above, we conclude that $\mathfrak{r}_{n+1}$ is also an ideal of $\mathfrak{q}$. This proves the inductive step and (2) follows.

(3a) and (3b) follow from the observation that, according to (2), the spaces $\mathfrak{r}_{1}, \ldots, \mathfrak{r}_{m}$ are invariant with respect to the transformations $S\left(X_{i}\right), i=1, \ldots, k$ (cf. [8]). Given (3a) and (3b), (3c) follows again from the observation that $\mathfrak{a}_{0 i}$ is invariant with respect to the algebra of linear transformations of $\mathfrak{q}$ generated by the transformations $S\left(X_{i}\right)$, $i=1, \ldots, k$.

We put $n=\operatorname{dim} \mathfrak{q}, \quad n_{0}=0$ and $n_{i}=\operatorname{dim}\left(\mathfrak{a}_{1} \oplus \cdots \oplus \mathfrak{a}_{i}\right), \quad i=$ $1, \ldots, m$. Then

$$
1 \leq k \leq n_{1}<\cdots<n_{m}=n .
$$

The choice of the basis of $\mathfrak{q}$. We assume now that $\mathfrak{q}$ is of type $\mathrm{R}$, i.e. that all the eigenvalues of the derivations ad $X, X \in \mathfrak{q}$ are purely imaginary (i.e. of the type $i a, a \in \mathbb{R}$ ).

Proposition 1.4. If $\mathfrak{q}$ is of type $R$, then there is a basis $\left\{X_{1}, \ldots\right.$, $\left.X_{n}\right\}$ of $\mathfrak{q}$ such that

(1) $X_{1}, \ldots, X_{k}$ are as in Proposition 1.1 and $X_{k+1}, \ldots, X_{n} \in \mathfrak{n}$,

(2) $\left\{X_{n_{t-1}+1}, \ldots, X_{n_{l}}\right\}$ is a basis of $\mathfrak{a}_{i}, i=1, \ldots, m$, 
(3) $\left\{X_{n_{t-1}+1}, \ldots, X_{n_{0 i}}\right\}$ and $\left\{X_{n_{0 i}+1}, \ldots, X_{n_{t}}\right\}$ are bases of $\mathfrak{a}_{0 i}$ and $\mathfrak{a}_{1 i}$ respectively, $i=1, \ldots, m$ and

(4) the number of the vectors $\left\{X_{n_{0 i}+1}, \ldots, X_{n_{i}}\right\}$ is even and they can be combined in pairs $\left\{X_{n_{0 t}+1}, X_{n_{0 t}+2}\right\}, \ldots,\left\{X_{j}, X_{j+1}\right\}, \ldots,\left\{X_{n_{t}-1}\right.$, $\left.X_{n_{t}}\right\}$ so that for every pair $\left\{X_{j}, X_{j+1}\right\}$ and every $l=1, \ldots, k$ there is $a_{l} \in \mathbb{R}$ such that

$$
\begin{aligned}
e^{S\left(X_{l}\right)} X_{j} & =\cos a_{l} X_{j}+\sin a_{l} X_{j+1} \\
e^{S\left(X_{l}\right)} X_{j+1} & =-\sin a_{l} X_{j}+\cos a_{l} X_{j+1}
\end{aligned}
$$

Proof. For $\left\{X_{n_{t-1}+1}, \ldots, X_{n_{0 l}}\right\}$ we choose any basis of $\mathfrak{a}_{0 i}$, so that (1) is satisfied. In order to choose $\left\{X_{n_{01}}+1, \ldots, X_{n_{i}}\right\}$ let us denote by $\mathfrak{a}_{1 i, \mathbb{C}}$ the complexification of $\mathfrak{a}_{1 i}$ and denote by $S\left(X_{j}\right)_{\mathbb{C}}$ the extension of $S\left(X_{j}\right)$ to $\mathfrak{a}_{1 i, \mathbb{C}}, i=1, \ldots, k$. Since $S\left(X_{j}\right)_{\mathbb{C}}$ is also semisimple, we can decompose $\mathfrak{a}_{1 i, \mathbb{C}}$ as $\mathfrak{a}_{1 i, b_{1}} \oplus \cdots \oplus \mathfrak{a}_{1 i, b_{h}}$ where $\mathfrak{a}_{1 i, b_{l}}=$ $\left\{Y \in \mathfrak{a}_{1 i, \mathbb{C}}, S\left(X_{j}\right)_{\mathbb{C}}(Y)=i b_{l} Y\right\}$ and $i b_{1}, \ldots, i b_{h} \in i \mathbb{R}$ are the different eigenvalues of $S\left(X_{j}\right)_{\mathbb{C}}$. Since $S\left(X_{l}\right)_{\mathbb{C}} S\left(X_{j}\right)_{\mathbb{C}}=S\left(X_{j}\right)_{\mathbb{C}} S\left(X_{l}\right)_{\mathbb{C}}$,

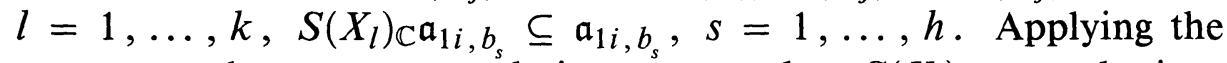
same procedure to $\mathfrak{a}_{1 i, b_{s}}$ relative to any other $S\left(X_{l}\right)_{\mathbb{C}}$, we obtain a decomposition

$$
\mathfrak{a}_{1 i, \mathbb{C}}=\mathfrak{b}_{1} \oplus \cdots \oplus \mathfrak{b}_{s}
$$

of $\mathfrak{a}_{1 i, \mathbb{C}}$ into $\left\{S\left(X_{j}\right)_{\mathbb{C}}, j=1, \ldots, k\right\}$-invariant subspaces, such that the linear tranformations induced in the $\mathfrak{b}_{l}$ by every $S\left(X_{j}\right)_{\mathbb{C}}$ are scalar multiplications by some $i a, a \in \mathbb{R}$. Moreover the subspaces $\mathfrak{b}_{l}$ can be taken to be one-dimensional. Let us identify $\mathfrak{a}_{1 i}, \mathbb{C}$ with $\{Z+$ $\left.i E, Z, E \in \mathfrak{a}_{1 i}\right\}$ and put $\bar{Y}=Z-i E, \operatorname{Re} Y=Z, \operatorname{Im} Y=E$ for $Y=Z+i E \in \mathfrak{a}_{1 i, \mathbb{C}}, Z, E \in \mathfrak{a}_{1 i}$ and $\bar{A}=\{\bar{Y}, Y \in A\}$ for $A \subseteq \mathfrak{a}_{1 i, \mathbb{C}}$. We observe that if ia, $a \in \mathbb{R}, a \neq 0$ is an eigenvalue of $S\left(X_{j}\right)_{\mathbb{C}}$ then $-i a$ is also an eigenvalue of the same multiplicity and that if $Y$ is an eigenvector for $i a, Y \neq 0$ then $\operatorname{Re} Y \neq 0, \operatorname{Im} Y \neq 0$, $\operatorname{Re} Y \neq \operatorname{Im} Y$ and $\bar{Y}$ is an eigenvector for the eigenvalue $-i a$. Using this observation we can easily see that the subspaces $\mathfrak{b}_{l}$ can be chosen in such a way, that the decomposition (1.5) can be written as

$$
\mathfrak{a}_{1 i, \mathbb{C}}=\mathfrak{b}_{i_{1}} \oplus \overline{\mathfrak{b}}_{i_{1}} \oplus \cdots \oplus \mathfrak{b}_{i_{r}} \oplus \overline{\mathfrak{b}}_{i_{r}}
$$

where $\mathfrak{b}_{l}=\left\{z Y_{l}, z \in \mathbb{C}\right\}$ for some $Y_{l} \in \mathfrak{a}_{1 i, \mathbb{C}}, Y_{l}=Z+i E, Z, E$ $\in \mathfrak{a}_{1 i}, Z \neq E, Z, E \neq 0$. 
We take $X_{n_{01}+1}=\operatorname{Re} Y_{j_{1}}, X_{n_{0 i}+2}=\operatorname{Im} Y_{j_{1}}, \ldots, X_{n_{i}-1}=\operatorname{Re} Y_{j_{r}}, X_{n_{i}}$ $=\operatorname{Im} Y_{j_{r}}$. We can easily see that the basis of $\mathfrak{q}$, constructed in this way, satisfies the requirements of the proposition.

2. The exponential coordinates of the second kind. Let $Q$ a simply connected solvable Lie group of polynomial growth and denote by $\mathfrak{q}$ its Lie algebra. According to a well-known theorem of Y. Guivarc'h [6], $\mathfrak{q}$ is of type $\mathrm{R}$, i.e. all the eigenvalues of the derivations ad $X(Y)=$ $[X, Y], X, Y \in \mathfrak{q}$ are of the type $i a, a \in \mathbb{R}$. We identify the elements of $\mathfrak{q}$ with the left invariant vector fields on $Q$.

The derivations $S(X), K(X), X \in \mathfrak{g}$ and the integers $n_{1}, \ldots, n_{m}$ are as in $\S 1$. We put

$$
\sigma(i)=j, \quad \text { if } n_{j-1}<i \leq n_{j}, \quad i=1, \ldots, n .
$$

We denote by $N$ the nil-radical of $Q$ i.e. the analytic subgroup of $Q$ having the nil-shadow $\mathfrak{n}$ of $\mathfrak{q}$ as its Lie algebra. Note that $N$ is nilpotent and that $Q / N$ is abelian.

Using the basis $\left\{X_{1}, \ldots, X_{n}\right\}$ of $\mathfrak{q}$ constructed in Proposition 1.4, we can consider the diffeomorphism

$$
\varphi: \mathbb{R}^{n} \rightarrow Q, \quad \varphi: x=\left(x_{n}, \ldots, x_{1}\right) \rightarrow \exp x_{n} X_{n} \cdots \exp x_{1} X_{1}
$$

which is called exponential coordinates of the second kind (cf. [12]).

We want to give an expression for $d \varphi^{-1}$. To this end, we shall need some notations.

We denote by $\overline{\mathrm{ad}} X_{i}$ and $\bar{K}\left(X_{i}\right)$ the linear transformations of $\mathfrak{q}$ defined by

$\overline{\operatorname{ad}}\left(X_{i}\right) X_{j}=0, \quad$ for $i \geq j$ and $\overline{\operatorname{ad}}\left(X_{i}\right) X_{j}=\operatorname{ad}\left(X_{i}\right) X_{j}, \quad$ for $i<j$, $\bar{K}\left(X_{i}\right) X_{j}=0, \quad$ for $i \geq j$ and $\bar{K}\left(X_{i}\right) X_{j}=K\left(X_{i}\right) X_{j}, \quad$ for $i<j$.

It follows from (1.1) and the fact that $S\left(X_{i}\right) X_{j}=0,1 \leq i, j \leq k$, that

$$
S\left(X_{i}\right) \bar{K}\left(X_{j}\right)=\bar{K}\left(X_{j}\right) S\left(X_{i}\right), \quad 1 \leq i, j \leq k .
$$

If $B(x)=b_{n}(x) \partial / \partial x_{n}+\cdots+b_{1}(x) \partial / \partial x_{1}$ is a vector field on $\mathbb{R}^{n}$, then we put $\operatorname{pr}_{i} B(x)=b_{i}(x)$. We also use the same notation for the left invariant vector fields on $Q$, i.e. if $E=c_{n} X_{n}+\cdots+c_{1} X_{1}$, then we put $\operatorname{pr}_{i} E=c_{i}$. 
Proposition 2.1. With the above notations we have

$$
\begin{aligned}
& \operatorname{pr}_{i} d \varphi^{-1} E(x)=\operatorname{pr}_{i}\left[e^{x_{n} \overline{\mathrm{ad}} X_{n}} \ldots e^{x_{1} \overline{\mathrm{ad}} X_{1}}\right](E) \operatorname{pr}_{i}\left[e^{x_{n} \bar{K}\left(X_{n}\right)} \cdots e^{x_{1} \bar{K}\left(X_{1}\right)} e^{x_{k} S\left(X_{k}\right)} \cdots e^{x_{1} S\left(X_{1}\right)}\right](E) \\
&=\operatorname{pr}_{i}\left\{\left[\begin{array}{c}
\sum_{\lambda_{1} \sigma(1)+\cdots+\lambda_{l-1} \sigma(i-1) \leq \sigma(i)-1} x_{1}^{\lambda_{1}} \cdots x_{i-1}^{\lambda_{t-1}} \\
\cdot \bar{K}^{\lambda_{i-1}}\left(X_{i-1}\right) \cdots \bar{K}^{\lambda_{1}}\left(X_{1}\right)
\end{array}\right] e^{x_{k} S\left(X_{k}\right)} \cdots e^{x_{1} S\left(X_{1}\right)}\right\}
\end{aligned}
$$

Proof. Clearly, the third equality in (2.2) is a more explicit version of the second one and the second equality follows immediately from the first one using (2.1). So it is enough to prove the first equality in (2.2).

Let $g=\exp x_{n} X_{n} \cdots \exp x_{1} X_{1} \in Q$ and $\gamma(t)=g \exp t E, t>0$ an integral curve of $E$. Then to prove the proposition it is enough to prove that

$$
\begin{aligned}
\gamma(t)= & \exp \left(x_{n}+t \mathrm{pr}_{n} e^{x_{n-1} \overline{\mathrm{ad}} X_{n-1}} \cdots e^{x_{1} \overline{\mathrm{ad}} X_{1}} E+O\left(t^{2}\right)\right) X_{n} \\
& \cdots \exp \left(x_{2}+t \mathrm{pr}_{2} e^{x_{1} \overline{\mathrm{ad}} X_{1}} E+O\left(t^{2}\right)\right) X_{2} \exp \left(x_{1}+t \mathrm{pr}_{1} E\right) X_{1} .
\end{aligned}
$$

(2.3) can be proved by induction on $n$ : It is trivially true for $n=1$. So assume that it is true for $n \leq l$. To prove that it is also true for $n=l+1$, observe that it follows from the Campell-Hausdorff formula that

$$
\begin{aligned}
\exp t E & =\exp t\left(c_{l+1} X_{l+1}+\cdots+c_{1} X_{1}\right) \\
& =\exp \left[\left(t c_{l+1}+O\left(t^{2}\right)\right) X_{l+1}+\cdots+\left(t c_{2}+O\left(t^{2}\right)\right) X_{2}\right] \exp c_{1} t X_{1} .
\end{aligned}
$$

Hence

$$
\begin{aligned}
& \gamma(t)=\exp x_{l+1} X_{l+1} \cdots \exp x_{1} X_{1} \\
& \cdot \exp \left[\left(t c_{l+1}+O\left(t^{2}\right)\right) X_{l+1}+\cdots+\left(t c_{2}+O\left(t^{2}\right)\right) X_{2}\right] \\
& \cdot \exp -x_{1} X_{1} \exp x_{1} X_{1} \exp t c_{1} X_{1} \\
& =\exp x_{l+1} X_{l+1} \cdots \exp x_{2} X_{2} \\
& \cdot \exp e^{x_{1} \overline{\mathrm{ad}} X_{1}}\left[\left(t c_{l+1}+O\left(t^{2}\right)\right) X_{l+1}+\cdots+\left(t c_{2}+O\left(t^{2}\right)\right) X_{2}\right] \\
& \cdot \exp \left(x_{1}+t c_{1}\right) X_{1} \text {. }
\end{aligned}
$$

Observing that the linear subspace of $\mathfrak{q}$ generated by the vectors $X_{l+1}, \ldots, X_{2}$ is in fact an ideal of the Lie algebra $q$ we can see that 
it follows from (2.4) and the inductive hypothesis that (2.3) is also true for $n=l+1$. This proves the inductive step and the proposition follows.

Let $Q_{N}$ be a simply connected nilpotent Lie group that admits the nil-shadow $\mathfrak{q}_{N}$ of $\mathfrak{q}$ (cf. $\S 1$ for the definition) as its Lie algebra. $Q_{N}$ is called the nil-shadow of $Q$.

We identify the elements of $\mathfrak{q}_{N}$ with the left invariant vector fields on $Q_{N}$ and if $X \in \mathfrak{q}$ then we denote by ${ }_{N} X$ the element of $\mathfrak{q}_{N}$ satisfying ${ }_{N} X(e)=X(e)$. We extend the transformations $S(X), X \in$ $\mathfrak{q}$, to $\mathfrak{q}_{N}$ by putting $S(X)_{N} Y={ }_{N}(S(X) Y)$.

Using again the exponential coordinates of the second kind

$$
\varphi_{N}: \mathbb{R}^{n} \rightarrow Q_{N}, \quad \varphi:\left(x_{n}, \ldots, x_{1}\right) \rightarrow \exp x_{n N} X_{n} \cdots \exp x_{1 N} X_{1}
$$

we can see that $Q_{N}$ is diffeomorphic with $\mathbb{R}^{n}$.

From now on, using the exponential coordinates of the second kind $\varphi$ and $\varphi_{N}$, we shall identify $Q$ and $Q_{N}$ as differential manifolds with $\mathbb{R}^{n}$.

It follows from (2.2) that if $x=\left(x_{n}, \ldots, x_{1}\right) \in \mathbb{R}^{n}$ and $E \in \mathfrak{q}$ then

$$
E(x)=\left(e^{x_{k} S\left(X_{k}\right)} \cdots e^{x_{1} S\left(X_{1}\right)}{ }_{N} E\right)(x) .
$$

3. The volume growth. Let $Q$ be a simply connected solvable Lie group of polynomial growth and $d g$ a left invariant Haar measure on $Q$.

We shall use the notations of $\S 2$. As it was explained in that section we identify $Q$ and $Q_{N}$ with $\mathbb{R}^{n}$.

Let $n_{0}, n_{1}, \ldots, n_{m}$ as in $\S 1$ and $\sigma(1), \ldots, \sigma(n)$ as in $\S 2$. We put

$$
d=\sigma(1)+\cdots+\sigma(n) .
$$

Let $E_{1}, \ldots, E_{p}$ as in Theorem 1, i.e. left invariant vector fields on $Q$ that satisfy Hörmander's condition. The control distance $d_{E}(\cdot, \cdot)$ associated to these vector fields is defined as follows (cf. [4], [14]):

We call an absolutely continuous path $\gamma:[0,1] \rightarrow Q$ admissible if and only if $\dot{\gamma}(t)=a_{1}(t) E_{1}+\cdots a_{p}(t) E_{p}$ for almost all $t \in[0,1]$. It is a consequence of the Hörmander condition that all points $x, y \in Q$ can be joint with at least one admissible path. We put $|\dot{\gamma}(t)|^{2}=a_{1}^{2}(t)+$ $\cdots+a_{p}^{2}(t)$ and we define

$$
\begin{aligned}
& d_{E}(x, y)=\inf \left\{\int_{0}^{1}|\dot{\gamma}(t)| d t, \gamma\right. \text { admissible path } \\
& \text { such that } \gamma(0)=x, \gamma(1)=y\} .
\end{aligned}
$$


We put $S_{E}(x, t)=\left\{y \in Q: d_{E}(x, y)<t\right\}, x \in Q, t>0$.

We want to describe the shape of the balls $S_{E}(e, t), t \geq 1$, and to estimate the $d g$-measure $\left(S_{E}(e, t)\right)$. To this end we shall need some notations. If $x=\left(x_{n}, \ldots, x_{1}\right)$, then we put

$$
\begin{gathered}
x_{t}=\left(t^{\sigma(n)} x_{n}, \ldots, t^{\sigma(1)} x_{1}\right), \quad t>0 . \\
D(x, t)=\left\{y=\left(\begin{array}{ll}
\left.y_{n}, \ldots, y_{1}\right) \in Q: \\
& \left.x_{i}-t^{\sigma(i)}<y_{i}<x_{i}+t^{\sigma(i)}, 1 \leq i \leq n\right\}, \quad t>0 .
\end{array}\right.\right.
\end{gathered}
$$

We also put $D_{t}=D(e, t)$ and $D=D(e, 1)$.

Proposition 3.1. Let $S_{E}(x, t)$ and $D_{t}$ be as above. Then there is $c>0$ such that

$$
\begin{gathered}
S_{E}\left(e, c^{-1} t\right) \subseteq D_{t} \subseteq S_{E}(e, c t), \quad t \geq 1, \\
c^{-1} t^{d} \leq d g \text {-measure }\left(S_{E}(e, t)\right) \leq c t^{d}, \quad t \geq 1 .
\end{gathered}
$$

Proof. As we see from $(0.1)$, the balls $S_{E}(e, t), t \geq 0$, behave, for large $t$, in the same way as the powers $V^{n}, n \in \mathbb{N}$, of a compact neighborhood $V$ of $e$. Hence the vector fields $\left\{E_{1}, \ldots, E_{p}\right\}$ can be replaced with the basis $\left\{X_{n}, \ldots, X_{1}\right\}$ of $\mathfrak{q}$. Furthermore, it follows from (2.5), that $\left\{X_{n}, \ldots, X_{1}\right\}$ can be replaced by $\left\{{ }_{N} X_{n}, \ldots,{ }_{N} X_{1}\right\}$ and then the proposition becomes a well-known result (cf. [5], [6], [15]).

Arguing in the same way as in the above proposition, we can prove the following lemma which we shall need later on.

LeMmA 3.2. Let $S_{E}(x, t), D(x, t)$ and $D$ be as above. Then there is $A>0$ and $\mu \in \mathbb{N}$ such that for all $x \in D, R \in(0,1]$ and $t>t_{0}=t_{0}(R)$, we have

$$
S_{E}\left(x_{t}, t R\right) \subseteq D\left(x_{t}, A t R^{1 / \mu}\right), \quad D\left(x_{t}, t R\right) \subseteq S_{E}\left(x_{t}, A t R^{1 / \mu}\right) .
$$

4. Generalization of some classical results of homogenization theory. Let $Q$ be a simply connected solvable Lie group of polynomial growth and $E_{1}, \ldots, E_{p}$ and $L$ as in Theorem 1, i.e. $E_{1}, \ldots, E_{p}$ are left invariant vector fields on $Q$ that satisfy Hörmander's condition; let $L=-\left(E_{1}^{2}+\cdots+E_{p}^{2}\right)$.

The purpose of this section is to generalize some classical results of the theory of homogenization (cf. [2]) in our context. In particular, we shall prove a homogenization formula for the operator $L$. The homogenized operator $L_{0}$ will be a left invariant sub-Laplacian defined 
on a limit group $Q_{H} \cdot Q_{H}$ is a homogeneous nilpotent Lie group and $L_{0}$ is invariant with respect to its dilation structure.

We fix a basis $\left\{X_{n}, \ldots, X_{1}\right\}$ of $\mathfrak{q}$, as in Proposition 1.4. As it was explained in $\S 2$, we identify $Q$ and $Q_{N}$ with $\mathbb{R}^{n}$.

$n_{0}, n_{1}, \ldots, n_{m}$ are as in $\S 1, \sigma(i), i=1, \ldots, n$, as in $\S 2$ and $D(x, t), D_{t}, D$ as in $\S 3$.

To simplify the notations, we shall use the summation convention for repeated indices.

The dilation. We denote by $\tau_{e}, \varepsilon>0$, the dilation of $\mathbb{R}^{n}$, hence of $Q$ and $Q_{N}$, defined by

$$
\tau_{\varepsilon}: \mathbb{R}^{n} \rightarrow \mathbb{R}^{n}, \quad \tau_{\varepsilon}:\left(x_{n}, \ldots, x_{1}\right) \rightarrow\left(\varepsilon^{\sigma(n)} x_{n}, \ldots, \varepsilon^{\sigma(1)} x_{1}\right) .
$$

We put

$$
\begin{aligned}
E_{\varepsilon, i} & =\frac{1}{\varepsilon} d \tau_{\varepsilon}\left(E_{i}\right), \quad i=1, \ldots, p \quad \text { and } \\
L_{\varepsilon} & =-\left(E_{\varepsilon, 1}^{2}+\cdots+E_{\varepsilon, p}^{2}\right), \quad 0<\varepsilon \leq 1 .
\end{aligned}
$$

The compactness. We recall the following Moser type Harnack inequality due to $\mathrm{N}$. Th. Varopoulos [13]:

TheOREM 4.1 (cf. N. Th. Varopoulos [13]). For all $a \in(0,1)$ there is a constant $c>0$ such that for all $t>0$ and $u \geq 0$ such that $L u=0$ in $S_{E}(x, t)$ we have

$$
\sup _{y \in S_{E}(x, a t)} u(y) \leq c \inf _{y \in S_{E}(x, a t)} u(y) .
$$

The above theorem provides a compactness on families of functions $u_{\varepsilon}$, satisfying

$$
\left\|u_{\varepsilon}\right\|_{\infty} \leq 1, \quad L_{\varepsilon} u_{\varepsilon}=0 \text { in } D, \quad 0<\varepsilon \leq 1 .
$$

More precisely we have the following

Proposition 4.2. Let $u_{\varepsilon}, 0<\varepsilon \leq 1$, be a family of functions satisfying (4.1). Then there is a subsequence, also denoted by $u_{\varepsilon}$, such that

$$
u_{\varepsilon} \rightarrow u_{0} \quad(\varepsilon \rightarrow 0)
$$

uniformly on the compact subsets of $D$.

Proof. The first thing to observe is that if $L_{\varepsilon} u_{\varepsilon}=0$ in $D$ then the function $u(x)=u\left(\tau_{\varepsilon}(x)\right)$ satisfies $L u=0$ in $D_{t}$, for $t=\varepsilon^{-1}$. Using 
this observation and Lemma 3.2, we can easily see that it follows from Theorem 4.1 that for every compact $U \subseteq D$ there are sequences

$$
\begin{aligned}
r_{1} & >r_{2}>\cdots, r_{i} \rightarrow 0(i \rightarrow \infty) \\
1 & >\varepsilon_{1}>\varepsilon_{2}>\cdots, \varepsilon_{i} \rightarrow 0(i \rightarrow \infty)
\end{aligned}
$$

and a constant $c>0$ such that

$$
\sup _{y \in D\left(x, r_{t+1}\right)} v_{\varepsilon}(y) \leq c \inf _{y \in D\left(x, r_{i+1}\right)} v_{\varepsilon}(y), \quad x \in U
$$

for all $v_{\varepsilon} \geq 0$ satisfying

$$
L_{\varepsilon} v_{\varepsilon}=0 \text { in } D\left(x, r_{i}\right), \quad \varepsilon \leq \varepsilon_{i} .
$$

Now, let $r_{i}$ such that $D\left(x, r_{i}\right) \subseteq D, x \in U, \varepsilon<\varepsilon_{i}, u_{\varepsilon}$ satisfying (4.1) and put

$$
\begin{gathered}
v_{\varepsilon}=1+u_{\varepsilon}, \\
M=\sup _{y \in D\left(x, r_{i+1}\right)} v_{\varepsilon}(y), \quad M^{\prime}=\sup _{y \in D\left(x, r_{i}\right)} v_{\varepsilon}(y), \\
m=\inf _{y \in D\left(x, r_{i+1}\right)} v_{\varepsilon}(y), \quad m^{\prime}=\inf _{y \in D\left(x, r_{i}\right)} v_{\varepsilon}(y) .
\end{gathered}
$$

Then it follows from (4.2) that

$$
\begin{aligned}
M^{\prime}-m & =\sup _{y \in D\left(x, r_{l+1}\right)}\left(M^{\prime}-v_{\varepsilon}(y)\right) \\
& \leq c \inf _{y \in D\left(x, r_{i+1}\right.}\left(M^{\prime}-v_{\varepsilon}(y)\right)=c\left(M^{\prime}-M\right), \\
M-m^{\prime} & =\sup _{y \in D\left(x, r_{t+1}\right)}\left(v_{\varepsilon}(y)-m^{\prime}\right) \\
& \leq c \inf _{y \in D\left(x, r_{i+1}\right.}\left(v_{\varepsilon}(y)-m^{\prime}\right)=c\left(m-m^{\prime}\right)
\end{aligned}
$$

and from this that

$$
M-m \leq \frac{c-1}{c+1}\left(M^{\prime}-m^{\prime}\right) .
$$

It follows from the above argument that for every compact $U \subseteq D$ and $\delta>0$ there is $r=r(U, \delta)>0$ and $\varepsilon_{0} \in(0,1)$ such that

$$
\left|u_{\varepsilon}(y)-u_{\varepsilon}(z)\right| \leq \delta, \quad y, z \in D(x, r), x \in U,
$$

for all $u_{\varepsilon}$ satisfying (4.1), with $\varepsilon \leq \varepsilon_{0}$ and the proposition follows by standard arguments. 
The limit group $Q_{H}$. Let $[\cdot, \cdot]_{N}$ as in $\S 1$ and $\mathfrak{r}_{1}, \ldots, \mathfrak{r}_{m}$ and $\mathfrak{a}_{1}, \ldots$, $\mathfrak{a}_{m}$ as in Proposition 1.2. Making use of the direct sum decomposition

$$
\mathfrak{q}=\mathfrak{a}_{1} \oplus \cdots \oplus \mathfrak{a}_{m}
$$

we consider the projection $\operatorname{pr}_{\mathfrak{a}_{i}}$ of $\mathfrak{q}$ on $\mathfrak{a}_{i}$.

We denote by $[\cdot, \cdot]_{H}$ the unique product on the linear space $q$ satisfying for $X \in \mathfrak{a}_{i}$ and $Y \in \mathfrak{a}_{j}$

$$
\begin{aligned}
& {[X, Y]_{H}=\operatorname{pr}_{\mathfrak{a}_{i+\jmath}}[X, Y]_{N}, \quad \text { if } i+j \leq m \text { and }} \\
& {[X, Y]_{H}=0, \quad \text { if } i+j>m .}
\end{aligned}
$$

It is easy to see that $[\cdot, \cdot]_{H}$ satisfies the Jacobi identity (observe that if $Z \in \mathfrak{a}_{h}$ and $X, Y$ are as above then it follows from the way the spaces $\mathfrak{r}_{i}, \mathfrak{a}_{i}, i=1, \ldots, m$, were defined that $\left[X,[Y, Z]_{H}\right]_{H}=$ $\left.\operatorname{pr}_{\mathfrak{a}_{l+j+h}}\left[X,[Y, Z]_{N}\right]_{N}\right)$. So, $\mathfrak{q}_{H}=\left(\mathfrak{q},[\cdot, \cdot]_{H}\right)$ is a nilpotent Lie algebra which is also stratified.

The limit group $Q_{H}$ is defined to be a simply connected Lie group that admits $\mathfrak{q}_{H}$ as its Lie algebra.

If $X \in \mathfrak{q}_{H}$ then we denote by ${ }_{H} X(e)$ the left invariant vector field on $Q_{H}$ satisfying ${ }_{H} X(e)=X(e)$ (e is the identity element of $\left.Q_{H}\right)$. Using the exponential coordinates of the second kind

$$
\varphi_{H}: \mathbb{R}^{n} \rightarrow Q_{H}, \quad \varphi:\left(x_{n}, \ldots, x_{1}\right) \rightarrow \exp x_{n H} X_{n} \cdots \exp x_{1 H} X_{1}
$$

we identify $Q_{H}$ with $\mathbb{R}^{n}$.

Having done this identification, we should notice that the family of dilations $\tau_{\varepsilon}, \varepsilon>0$, introduced in the beginning of this section, is exactly the natural family of dilations which is compatible with the Lie group structure of $Q_{H}$ (cf. [5]).

The coefficients of the operator $L$. Let us fix a vector field $E_{h}$, $1 \leq h \leq p$. Then from (2.2) and with the same notations we have that

$$
E_{h}=\left(a_{n}^{h}+b_{n}^{h}\right) \frac{\partial}{\partial x_{n}}+\cdots+\left(a_{1}^{h}+b_{1}^{h}\right) \frac{\partial}{\partial x_{1}}
$$

where

$$
a_{i}^{h}(x)=\alpha_{i}^{h}(x, x), \quad b_{i}^{h}(x)=\beta_{i}^{h}(x, x),
$$




$$
\begin{aligned}
& \alpha_{i}^{h}(x, y)=\operatorname{pr}_{i}\left\{\left[\sum_{\lambda_{1} \sigma(1)+\cdots+\lambda_{t-1} \sigma(i-1)=\sigma(i)-1} x_{1}^{\lambda_{1}} \cdots x_{i-1}^{\lambda_{i-1}}\right.\right. \\
& \left.\cdot \bar{K}^{\lambda_{i-1}}\left(X_{i-1}\right) \cdots \bar{K}^{\lambda_{1}}\left(X_{1}\right)\right] \\
& \left.\cdot e^{y_{k} S\left(X_{k}\right)} \cdots e^{y_{1} S\left(X_{1}\right)}\right\}\left(E_{h}\right)
\end{aligned}
$$

and

(4.4)

$$
\begin{gathered}
\beta_{i}^{h}(x, y)=\operatorname{pr}_{i}\left\{\left[\sum_{\lambda_{1} \sigma(1)+\cdots+\lambda_{t-1} \sigma(i-1)<\sigma(i)-1} x_{1}^{\lambda_{1}} \cdots x_{i-1}^{\lambda_{t-1}}\right.\right. \\
\left.\cdot \bar{K}^{\lambda_{i-1}}\left(X_{i-1}\right) \cdots \bar{K}^{\lambda_{1}}\left(X_{1}\right)\right] \\
\cdot e^{\left.y_{k} S\left(X_{k}\right) \cdots e^{y_{1} S\left(X_{1}\right)}\right\}\left(E_{h}\right),} \\
x=\left(x_{n}, \ldots, x_{1}\right), y=\left(y_{n}, \ldots, y_{1}\right), \quad x, y \in \mathbb{R}^{n}, 1 \leq i \leq n .
\end{gathered}
$$

We have the following proposition which is a direct consequence of the above definitions and the way the vectors $X_{1}, \ldots, X_{n}$ were chosen (cf. Propositions 1.3).

Proposition 4.3. The coefficients $\alpha_{i}^{h}(x, y)$ and $\beta_{i}^{h}(x, y)$ have the following properties:

(1) $\alpha_{i}^{h}(x, y)=$ constant, for $1 \leq i \leq k$,

(2) if $k<i \leq n_{1}$, then $\alpha_{i}^{h}(x, y)=\alpha_{i}^{h}(y)$ and it is periodic with respect to $y$,

(3) if $n_{1}<i \leq n$, then $\alpha_{i}^{h}(x, y)$ and $\beta_{i}^{h}(x, y)$ can be written as finite sums of terms of the form $p(x) \varphi(y)$, where $p(x)=c x_{i_{1}} \cdots x_{i_{l}}$, $c \in \mathbb{R}, 1 \leq i_{j}<i, 1 \leq j \leq l$ and $\varphi(y)=\cos a y_{j}$ or $\sin a y_{j}$ for some $1 \leq j \leq k$, hence a periodic function and

(4) $\beta_{i}^{h}(x, y)=0,1 \leq i \leq n_{1}$.

Let $\bar{K}_{H}\left(X_{i}\right), 1 \leq i \leq n$, be the linear transformations of $\mathfrak{q}$ defined by

$\bar{K}_{H}\left(X_{i}\right) X_{j}=0, \quad j \leq i, \quad$ and $\quad \bar{K}_{H}\left(X_{i}\right) X_{j}=\left[X_{i}, X_{j}\right]_{H}, \quad i \leq j$. 
Then (4.3) becomes

$$
\alpha_{i}^{h}(x, y)=\operatorname{pr}_{i}\left[e^{x_{i-1} \bar{K}_{H}\left(X_{t-1}\right)} \cdots e^{x_{1} \bar{K}_{H}\left(X_{1}\right)} e^{y_{k} S\left(X_{k}\right)} \cdots e^{y_{1} S\left(X_{1}\right)}\right]\left(E_{h}\right)
$$

and from this we have

$$
\alpha_{i}^{h}(x, y)=\sum_{1 \leq j \leq n_{1}} \alpha_{j}^{h}(y) \operatorname{pr}_{i}\left[e^{x_{i-1} \bar{K}_{H}\left(X_{i-1}\right)} \cdots e^{x_{1} \bar{K}_{H}\left(X_{1}\right)}\right]\left(X_{j}\right) .
$$

Let us put, for $1 \leq i, j \leq n$

$$
\begin{aligned}
& \alpha_{i j}(x, y)= \sum_{1 \leq h \leq p} \alpha_{i}^{h}(x, y) \alpha_{j}^{h}(x, y), \\
& \beta_{i j}(x, y)=\sum_{1 \leq h \leq p}\left[\alpha_{i}^{h}(x, y) \beta_{j}^{h}(x, y)+\beta_{i}^{h}(x, y) \beta_{j}^{h}(x, y)\right. \\
&\left.+\beta_{i}^{h}(x, y) \alpha_{j}^{h}(x, y)\right] \\
& a_{i j}(x)=\alpha_{i j}(x, x), \quad b_{i j}(x)=\beta_{i j}(x, x) .
\end{aligned}
$$

Then we have (we use the summation convention for repeated indices)

$$
L=A+B, \quad \text { where } A=-\frac{\partial}{\partial x_{i}} a_{i j}(x) \frac{\partial}{\partial x_{j}} \text { and } B=-\frac{\partial}{\partial x_{i}} b_{i j}(x) \frac{\partial}{\partial x_{j}} \text {. }
$$

In the following proposition we have gathered some properties of the coefficients $\alpha_{i j}(x, y)$ and $\beta_{i j}(x, y)$ which are immediate consequences of the definitions.

Proposition 4.4. (1) The coefficients $\alpha_{i j}(x, y)$ and $\beta_{i j}(x, y)$ are finite sums of terms of the form $p(x) \varphi(y)$, where $p(x)=c x_{i_{1}} \cdots x_{i_{l}}$, $c \in \mathbb{R}, 1 \leq i_{h}<\max (i, j), 1 \leq h \leq l$, and $\varphi(y)=\cos a y_{j}$ or $\sin a y_{j}$ for some $1 \leq j \leq k$, hence a periodic function.

(2) $\alpha_{i j}(x, y)=\alpha_{i j}(y), 1 \leq i, j \leq n_{1}$.

(3) $\alpha_{i j}(x, y)=$ constant, $1 \leq i, j \leq k$.

(4) $\beta_{i j}(x, y)=0,-1 \leq i, j \leq n_{1}$.

The correctors. We put

$$
A(x)=-\frac{\partial}{\partial y_{i}} \alpha_{i j}(x, y) \frac{\partial}{\partial y_{j}} \text {. }
$$

If $f(x, y)$ is a finite sum of functions periodic with respect to the variable $y$ then we denote by $\mathfrak{M}(f)(x)$ the mean of $f$, defined by

$$
\mathfrak{M}(f)(x)=\lim _{t \rightarrow \infty} \frac{1}{\left|D_{t}\right|} \int_{D_{t}} f(x, y) d y
$$

where $\left|D_{t}\right|$ denotes the volume of $D_{t}$. 
The correctors $\chi^{j}(x, y), 1 \leq j \leq n$, are defined to be $C^{\infty}$ functions satisfying

$$
A(x) \chi^{j}(x, y)=-\frac{\partial}{\partial y_{i}} \alpha_{i j}(x, y), \quad \mathfrak{M}\left(\chi^{j}\right)=0 .
$$

They are defined as follows:

For $1 \leq j \leq n_{1}$ they are defined to be the unique solutions of the problem

$$
A(x) \chi^{j}(x, y)=-\frac{\partial}{\partial y_{i}} \alpha_{i j}(x, y), \quad \mathfrak{M}\left(\chi^{j}\right)=0 .
$$

Notice that, in view of Proposition 4.4,

$$
\sum_{1 \leq i \leq n} \frac{\partial}{\partial y_{i}} \alpha_{i j}(x, y)=\sum_{1 \leq i \leq k} \frac{\partial}{\partial y_{i}} \alpha_{i j}\left(y_{k}, \ldots, y_{1}\right), \quad 1 \leq j \leq n_{1}
$$

which is a periodic function with mean zero and therefore the correctors $\chi^{j}, 1 \leq j \leq n_{1}$, are well defined.

For $n_{1}<j \leq n$ the correctors $\chi^{j}$ are defined by

$$
\chi^{j}(x, y)=\sum_{1 \leq l \leq n_{1}} \chi^{l}(y) \operatorname{pr}_{j}\left[e^{x_{j-1} \bar{K}_{H}\left(X_{j-1}\right)} \cdots e^{x_{1} \bar{K}_{H}\left(X_{1}\right)}\right]\left(X_{l}\right) .
$$

An immediate consequence of the definition is the following

Proposition 4.5. (1) $A(x)\left(\chi^{j}(x, y)-y_{j}\right)=0,1 \leq j \leq n$.

(2) $\chi^{j}(x, y)=\chi^{j}\left(x,\left(y_{k}, \ldots, y_{1}\right)\right), 1 \leq j \leq n$.

(3) $\chi^{j}=0,1 \leq j \leq k$.

(4) If $k<j \leq n_{1}$, then $\chi^{j}(x, y)=\chi^{j}(y)$ and is periodic with respect to $y$.

The homogenized operator $L_{0}$. We put

$$
q_{i j}(x)=\mathfrak{M}\left\{\alpha_{i j}(x, y)-\alpha_{i l}(x, y) \frac{\partial}{\partial y_{l}} \chi^{j}(x, y)\right\}
$$

The homogenized operator $L_{0}$ is defined by

$$
L_{0}=-\frac{\partial}{\partial x_{i}} q_{i j}(x) \frac{\partial}{\partial x_{j}} \text {. }
$$

Proposition 4.6. (1) $q_{i j}(x)=q_{j i}(x), 1 \leq i, j \leq n$.

(2) $q_{i j}(x)=$ constant $, 1 \leq i, j \leq n_{1}$. 
(3)

$$
\begin{aligned}
q_{i j}(x)=\sum_{1 \leq l, \mu \leq n_{1}}\left\{\operatorname{pr}_{i}\left[e^{x_{i-1} \bar{K}_{H}\left(X_{i-1}\right)} \cdots e^{x_{1} \bar{K}_{H}\left(X_{1}\right)}\right]\left(X_{l}\right)\right\} q_{l \mu} \\
\cdot\left\{\operatorname{pr}_{j}\left[e^{x_{j-1} \bar{K}_{H}\left(X_{j-1}\right)} \cdots e^{x_{1} \bar{K}_{H}\left(X_{1}\right)}\right]\left(X_{\mu}\right)\right\}, \quad 1 \leq i, j \leq n .
\end{aligned}
$$

Proof. (2) and (3) follow from the definitions and Propositions 4.4 and 4.5. To prove (1) let us observe that

$$
q_{i j}(x)=\mathfrak{M}\left\{\left(\frac{\partial}{\partial y_{h}} y_{i}\right) \alpha_{h l}(x, y) \frac{\partial}{\partial y_{l}}\left[y_{j}-\chi^{j}(x, y)\right]\right\}
$$

and that from the definition of the correctors $\chi^{j}, 1 \leq j \leq n$, we have that

$$
\mathfrak{M}\left\{\left[\frac{\partial}{\partial y_{h}} \chi^{i}(x, y)\right] \alpha_{h l}(x, y) \frac{\partial}{\partial y_{l}}\left[y_{j}-\chi^{j}(x, y)\right]\right\}=0 .
$$

Hence

$$
\text { (4.8) } q_{i j}(x)=\mathfrak{M}\left\{\frac{\partial}{\partial y_{h}}\left[y_{i}-\chi^{i}(x, y)\right] \alpha_{h l}(x, y) \frac{\partial}{\partial y_{l}}\left[y_{j}-\chi^{j}(x, y)\right]\right\}
$$

and the proposition follows.

LEMMA 4.7. The operator

$$
L_{0}^{\prime}=-\sum_{1 \leq i, j \leq n_{1}} \frac{\partial}{\partial x_{i}} q_{i j}(x) \frac{\partial}{\partial x_{j}}
$$

is an elliptic operator with constant coefficients in $\mathbb{R}^{n_{1}}$.

Proof. Let $\xi=\left(\xi_{1}, \ldots, \xi_{n_{1}}\right) \in \mathbb{R}^{n_{1}}, \xi \neq 0$, and (cf. Proposition 4.5)

$$
f(y)=\xi_{1}\left[y_{1}-\chi^{1}(y)\right]+\cdots+\xi_{n_{1}}\left[y_{n_{1}}-\chi^{n_{1}}(y)\right] .
$$

Then, from (4.7) we have that

$$
\sum_{1 \leq i, j \leq n_{1}} q_{i j} \xi_{i} \xi_{j}=\mathfrak{M}\left\{\left[\frac{\partial}{\partial y_{h}} f(y)\right] \alpha_{h l}(y) \frac{\partial}{\partial y_{l}} f(y)\right\}
$$

and from Proposition 4.4 that

$$
\mathfrak{M}\left\{\left[\frac{\partial}{\partial y_{l}} f(y)\right] \alpha_{l \mu}(y) \frac{\partial}{\partial y_{\mu}} f(y)\right\}=\mathfrak{M}\left\{\left(E_{1} f\right)^{2}+\cdots+\left(E_{p} f\right)^{2}\right\} .
$$

So to prove the lemma it is enough to prove that

$$
\mathfrak{M}\left\{\left(E_{1} f\right)^{2}+\cdots+\left(E_{p} f\right)^{2}\right\} \neq 0 .
$$


To do this, since the function $\left(E_{1} f\right)^{2}+\cdots+\left(E_{p} f\right)^{2}$ is a finite sum of $C^{\infty}$ periodic functions, it is enough to prove that there is an open set $U \subseteq \mathbb{R}^{n}$ and $1 \leq i \leq p$ such that $E_{i} f(y) \neq 0, y \in U$. This follows from the observation that if $E_{i} f(y)=0, \forall y \in \mathbb{R}^{n}$ then, since the vector fields $E_{1}, \ldots, E_{p}$ satisfy Hörmander's condition, we would have that $f(y)=c, \forall y \in \mathbb{R}^{n}$ and hence that

$$
\xi_{1} y_{1}+\cdots+\xi_{n_{1}} y_{n_{1}}=\xi_{1} \chi^{1}(y)+\cdots+\xi_{n_{1}} \chi^{n_{1}}(y)+c
$$

which is absurd since the second member of the above equality is a sum of periodic functions.

It follows from the above proposition that there are linearly independent vector fields $Y_{1}, \ldots, Y_{n_{1}}$ in $\mathbb{R}^{n_{1}}$, with constant coefficients, such that $L_{0}^{\prime}=-\left(Y_{1}^{2}+\cdots+Y_{n_{1}}^{2}\right)$. Let us denote by $W_{1}, \ldots, W_{n_{1}}$, respectively the images of $Y_{1}, \ldots, Y_{n_{1}}$ under the linear isomorphism of $\mathbb{R}^{n_{1}}$ with $\mathfrak{a}_{1}$ that maps $\partial / \partial x_{i} \rightarrow X_{i}, 1 \leq i \leq n_{1}$, and denote by ${ }_{H} W_{1}, \ldots,{ }_{H} W_{n_{1}}$ the left invariant vector fields on the limit group $Q_{H}$ satisfying ${ }_{H} W_{i}(e)=W_{i}, i=1, \ldots, n_{1}$. Since $Q_{H}$ (as well as $Q$ ) has been identified as differential manifold with $\mathbb{R}^{n},{ }_{H} W_{1}, \ldots,{ }_{H} W_{n-1}$ can also be viewed as vector fields on $\mathbb{R}^{n}$ (as well as on $Q$ ). Then it follows from Proposition 4.6(3) that the limit operator $L_{0}$ satisfies

$$
L_{0}=-\frac{\partial}{\partial x_{i}} q_{i j}(x) \frac{\partial}{\partial x_{j}}=-\left(W_{1}^{2}+\cdots+W_{n_{1}}^{2}\right),
$$

i.e. $L_{0}$ is a left invariant sub-Laplacian on $Q_{H}$, which is also invariant with respect to the natural dilation structure of $Q_{H}$ (cf. [5]).

The homogenization formula. Now we can state the following

Proposition 4.8. Let $u_{0}$ be as in Proposition 4.2 and $L_{0}$ as above. Then

$$
L_{0} u_{0}=0 \text { in } D \text {. }
$$

The proof of the above proposition is exactly the same with the proof of the homogenization formula in the classical case of uniformly elliptic second order differential operators with periodic coefficients (cf. [2]).

The only modification is that, since in our case we deal with hypoelliptic and not uniformly elliptic operators we have to replace $D$ with a neighborhood $U$ of 0 which is very regular, in the sense of Bony [4], i.e. it is such that

(i) $U=B_{1} \cap B_{2}$, where $B_{1}$ and $B_{2}$ are two Euclidean balls of $\mathbb{R}^{n}$ and 
(ii) if $x \in \partial U$, hence $x \in B_{i}$ for some $i \in\{1,2\}, v=\left(v_{n}, \ldots, v_{1}\right)$ is the vertical unit vector to the ball $B_{i}$ at the point $x$ and the operators $L_{\varepsilon}, 0<\varepsilon \leq 1$, are written in divergence form as $L_{\varepsilon}=$ $-\left(\partial / \partial x_{i}\right) a_{i j}^{\varepsilon}\left(\partial / \partial x_{j}\right)$ then

$$
\sum_{1 \leq i, j \leq n} a_{i j}^{\varepsilon}(x) v_{i} v_{j}>0
$$

Observe that since $D$ can be scaled down to a subset of $U$, we can indeed replace it by $U$.

To see that not only 0 but every $y=\left(y_{n}, \ldots, y_{1}\right) \in \mathbb{O}$ has such a very regular neighborhood $U$ let us observe that $a_{i i j}^{\varepsilon}=$ const., $1 \leq i$, $j \leq k$. Hence, if $\xi \neq 0, \xi=\left(\xi_{n}, \ldots, \xi_{1}\right), \xi_{k+1}=\cdots=\xi_{n}=0$, then

$$
\sum_{1 \leq i, j \leq n} a_{i j}^{\varepsilon} \xi_{i} \xi_{j}>0, \quad 0<\varepsilon \leq 1
$$

So the intersection $U=B_{1} \cap B_{2}$ of the balls $B_{1}$ and $B_{2}$ of radius $M+\delta$, centered at the points $y+M \xi$ and $y-M \xi$ respectively, for $M$ large and $\delta$ small enough is a very regular neighborhood of $y$.

Apart from this modification the energy proof of the homogenization formula (cf. [2]) can be carried through without any change at all.

5. The proof of Theorem 1. The proof of Theorem 1 will be based on a rescaling argument of $M$. Avellaneda and F. H. Lin [1] that we shall adapt in our context.

We shall use the notations of $\S 4$.

LeMMA 5.1. For all $\mu \in(0,1)$ there are $\theta \in(0,1), \varepsilon_{0} \in(0,1)$ and $c>0$ such that for all $0<\varepsilon \leq \varepsilon_{0}$ and all functions $u_{\varepsilon}$ satisfying

$$
L_{\varepsilon} u_{\varepsilon}=0 \quad \text { in } D, \quad\left\|u_{\varepsilon}\right\|_{\infty} \leq 1
$$

we have that

$$
\sup _{x \in D_{\theta}}\left|u_{\varepsilon}(x)-A_{0}^{\varepsilon}-\sum_{1 \leq j \leq n_{1}} A_{j}^{\varepsilon}\left(x_{j}-\varepsilon \chi^{j}\left(\tau_{\varepsilon^{-1}} x\right)\right)\right|<\theta^{1+\mu}
$$

where, $A_{j}^{\varepsilon}, 0 \leq j \leq n_{1}$, are constants satisfying $\left|A_{j}^{\varepsilon}\right|<c, 0 \leq j \leq n_{1}$.

Proof. First we observe that there is $\mu^{\prime}>\mu, \theta \in(0,1)$ and $c>0$ such that for all $u$ satisfying

$$
L_{0} u=0 \quad \text { in } D, \quad\|u\|_{\infty} \leq 1
$$


we have that

$$
\sup _{x \in D_{\theta}} \mid\left(u(x)-A_{0}^{0}-\sum_{1 \leq j \leq n_{1}} x_{j} \mid<\theta^{1+\mu^{\prime}}\right.
$$

where $A_{j}^{0}, 0 \leq j \leq n_{1}$, are constants satisfying $\left|A_{j}^{0}\right|<c, 0 \leq j \leq$ $n_{1}$. This follows from the fact that the homogenized operator $L_{0}$ is hypoelliptic (cf. [4]).

Let us fix these values of $\theta$ and $c$. If (5.1) weren't true then there would be a sequence of functions $u_{\varepsilon_{m}}, \varepsilon_{m} \rightarrow 0(m \rightarrow \infty)$ not satisfying (5.1). We can assume, by extracting a subsequence if necessary, that $u_{\varepsilon_{m}} \rightarrow u_{0} \quad(m \rightarrow \infty)$ uniformly on the compact subsets of $D$, and then $u$ would satisfy (5.2).

Let us take $A_{j}^{\varepsilon_{m}}=A_{j}^{0}, 0 \leq j \leq n_{1}$. Then using the assumption that the functions $u_{\varepsilon_{m}}$ do not satisfy $(5.1)$ and passing to the limit we have that

$$
\theta^{1+\mu}<\sup _{x \in D_{\theta}}\left|u(x)-A_{0}^{0}-\sum_{1 \leq j \leq n_{1}} A_{j}^{0} x_{j}\right|<\theta^{1+\mu^{\prime}}
$$

which is absurd. Hence the lemma.

LEMMA 5.2. Let $\theta, \mu$ and $\varepsilon_{0}$ be as in Lemma 5.1. Then there is a constant $c>0$ such that for all $m \in \mathbb{N}$ and $\varepsilon \in(-1,1)$ such that $\varepsilon \leq \theta^{m-1} \varepsilon_{0}$ and all $u_{\varepsilon}$ satisfying

$$
L_{\varepsilon} u_{\varepsilon}=0 \quad \text { in } D, \quad\left\|u_{\varepsilon}\right\|_{\infty} \leq 1
$$

we have that

$$
\sup _{x \in D_{\theta^{m}}}\left|u_{\varepsilon} x-A_{0}^{\varepsilon, m}-\sum_{1 \leq j \leq n_{1}} A_{j}^{\varepsilon, m}\left(x_{j}-\varepsilon \chi^{j}\left(\tau_{\varepsilon^{-1}} x\right)\right)\right|<\theta^{m(1+\mu)}
$$

where $A_{j}^{\varepsilon, m}, 0 \leq j \leq n_{1}$, are constants satisfying $\left|A_{j}^{\varepsilon, m}\right|<c, 0 \leq$ $j \leq n_{1}$.

Proof. The lemma will be proved by induction. For $m=1$ we are in the case of Lemma 5.1. So assume that (5.3) is true for some $m \in \mathbb{N}$. We put

$$
\begin{aligned}
& \text { (5.4) } w_{\varepsilon}(x)=\theta^{m(1+\mu)}\left[u_{\varepsilon}\left(\tau_{\theta^{m}} x\right)-A_{0}^{\varepsilon, m}\right. \\
& \left.-\sum_{1 \leq j \leq n_{1}} A_{j}^{\varepsilon, m}\left(\theta^{m} x_{j}-\varepsilon \chi^{j}\left(\tau_{\varepsilon^{-1} \theta^{m}} x\right)\right)\right] .
\end{aligned}
$$


Then we have that

$$
L_{\varepsilon^{\theta-m}} w_{\varepsilon}=0 \quad \text { in } D, \quad\left\|w_{\varepsilon}\right\|_{\infty} \leq 1
$$

Therefore it follows from Lemma 5.1 that, for $\varepsilon \theta^{-m} \leq \varepsilon_{0}$ we have that

(5.5) $\sup _{x \in D_{\theta}}\left|w_{\varepsilon}(x)-B_{0}^{\varepsilon}-\sum_{1 \leq j \leq n_{1}} B_{j}^{\varepsilon}\left(\theta^{m} x_{j}-\varepsilon \theta^{-m} \chi^{j}\left(\tau_{\varepsilon^{-1}} \theta^{m} x\right)\right)\right|<\theta^{1+\mu}$ with $\left|B_{j}^{\varepsilon}\right|<c, 0 \leq j \leq n_{1}$ (the constant $c$ being as in Lemma 5.1).

Let us put

$$
\begin{aligned}
& A_{0}^{\varepsilon, m+1}=A_{0}^{\varepsilon, m}+\theta^{m(1+\mu)} B_{0}^{\varepsilon}, \\
& A_{j}^{\varepsilon, m+1}=A_{j}^{\varepsilon, m}+\theta^{m \mu} B_{j}^{\varepsilon}, \quad 1 \leq j \leq n_{1} .
\end{aligned}
$$

Then putting (5.4) and (5.5) together we have that

$$
\begin{aligned}
\sup _{x \in D_{\theta}} \theta^{-m(1+\mu)} \mid & u_{\varepsilon}\left(\tau_{\theta^{m}} x\right)-A_{0}^{\varepsilon, m+1} \\
& \quad-\sum_{1 \leq j \leq n_{1}} A_{j}^{\varepsilon, m+1}\left(\theta^{m} x_{j}-\varepsilon \chi^{j}\left(\tau_{\varepsilon^{-1} \theta^{m}} x\right)\right) \mid<\theta^{1+\mu}
\end{aligned}
$$

and from this

$$
\begin{aligned}
\sup _{x \in D_{\theta^{m+1}}} \mid u_{\varepsilon}(x)-A_{0}^{\varepsilon, m+1} & \\
& -\sum_{1 \leq j \leq n_{1}} A_{j}^{\varepsilon, m+1}\left(x_{j}-\varepsilon \chi^{j}\left(\tau_{\varepsilon^{-1}} x\right)\right) \mid<\theta^{(m+1)(1+\mu)}
\end{aligned}
$$

which proves the inductive step and the lemma follows.

Corollary 5.3. Let $\varepsilon_{0}$ be as in Lemma 5.2. Then there is $c>0$ such that for all $\varepsilon \in\left(0, \varepsilon_{0}\right]$ and all $u_{\varepsilon}$ satisfying

$$
L_{\varepsilon} u_{\varepsilon}=0 \quad \text { in } D, \quad\left\|u_{\varepsilon}\right\|_{\infty} \leq 1
$$

we have that

$$
\sup _{x \in D_{\varepsilon / \varepsilon_{0}}}\left|u_{\varepsilon}(x)-A_{0}^{\varepsilon}\right|<c \frac{\varepsilon}{\varepsilon_{0}}
$$

where $A_{0}^{\varepsilon}$ is a constant such that $A_{0}^{\varepsilon}<c$. 
COROLlary 5.4. There is a constant $c>0$ such that for all $u$ satisfying

$$
L u=0 \quad \text { in } D_{t}, \quad t \geq 1
$$

we have that

$$
\sup _{x \in D}\left|u(x)-A_{0}\right|<\frac{c}{t}\|u\|_{\infty}
$$

where $A_{0}$ is a constant such that $\left|A_{0}\right|<c\|u\|_{\infty}$.

Proof. The corollary follows from Corollary 5.3 and the observation that if $u$ satisfies

$$
L u=0 \quad \text { in } D_{t}, \quad t \geq 1
$$

then the function $u_{\varepsilon}$ defined by $u_{\varepsilon}(x)=u\left(\tau_{t} x\right), \varepsilon=1 / t$ satisfies

$$
L_{\varepsilon} u_{\varepsilon}=0 \text { in } D \text {. }
$$

Proof of Theorem 1. It is enough to prove Theorem 1 when $Q$ is simply connected. In that case it is an immediate consequence of Corollary 5.4 and Theorem 4.1 .

\section{REFERENCES}

[1] M. Avellaneda and F. H. Lin, Compactness methods in the theory of homogenization, Comm. Pure Appl. Math., 40 (1987), 803-847.

[2] A. Bensoussan, J. L. Lions and G. Papanicolaou, Asymptotic Analysis of Periodic Structures, North-Holland, Amsterdam, 1978.

[3] A. S. Besicovitch, Almost Periodic Functions, Dover Publications, 1954.

[4] J. M. Bony, Principe du maximum, inégalité de Harnack et unicité du problème de Cauchy pour les opérateurs élliptiques dégénérés, Ann. Inst. Fourier, 19 (1), (1969), 277-304.

[5] G. B. Folland and E. Stein, Hardy Spaces on Homogeneous Groups, Princeton University Press, 1982.

[6] Y. Guivarc'h, Croissance polynômiale et périodes de fonctions harmoniques, Bull. Sci. Math. France, 101 (1973), 149-152.

[7] L. Hörmander, Hypoelliptic second order operators, Acta Math., 119 (1967), 147-171.

[8] N. Jacobson, Lie Algebras, Wiley, 1962.

[9] J. Moser, On Harnack's theorem for elliptic differential equations, Comm. Pure Appl. Math., 17 (1964), 577-591.

[10] E. Stein, Singular integrals and differentiability properties of functions, Ann. of Math. Studies, 1970.

[11] _ Topics in Harmonic Analysis, Princeton University Press, 1970.

[12] V. S. Varadarajan, Lie Groups, Lie Algebras and Their Representations, SpringerVerlag, 1984. 
[13] N. Th. Varopoulos, Fonctions harmoniques positives sur les groupes de Lie, C.R. Acad. Sci. Paris, 309, serie I, (1987), 519-521.

[14] __ Analysis on Lie groups, J. Funct. Anal., 76 no. 2, (1988), 346-410.

[15] N. Th. Varopoulos, L. Saloff-Coste and T. Coulhon, Harmonic Analysis on Lie Groups, Cambridge University Press, to appear.

Received February 27, 1990 and in revised form February 24, 1992.

Université De PARIS-SUd

Mathématiques, BÂT. 425

91405 Orsay Cedex

FRANCE 



\section{PACIFIC JOURNAL OF MATHEMATICS}

Founded by

E. F. BECKENBACH (1906-1982) F. Wolf (1904-1989)

\section{EDITORS}

\author{
V. S. VARADARAJAN \\ (Managing Editor) \\ University of California \\ Los Angeles, CA 90024-1555 \\ vsv@math.ucla.edu \\ F. Michael Christ \\ University of California \\ Los Angeles, CA 90024-1555 \\ christ@math.ucla.edu
}

\section{Herbert Clemens}

University of Utah

Salt Lake City, UT 84112

clemens@math.utah.edu

\author{
ThOMAs ENRIGHT \\ University of California, San Diego \\ La Jolla, CA 92093 \\ tenright@ucsd.edu \\ Nicholas Ercolani \\ University of Arizona \\ Tucson, AZ 85721 \\ ercolani@math.arizona.edu \\ R. FINN \\ Stanford University \\ Stanford, CA 94305 \\ finn@gauss.stanford.edu \\ VAUGHAN F. R. Jones \\ University of California \\ Berkeley, CA 94720 \\ vfr@math.berkeley.edu
}

STEVEN KeRCKHOFF

Stanford University

Stanford, CA 94305

spk@gauss.stanford.edu

Martin ScharlemanN

University of California

Santa Barbara, CA 93106

mgscharl@henri.ucsb.edu

Harold Stark

University of California, San Diego

La Jolla, CA 92093

\section{SUPPORTING INSTITUTIONS}

UNIVERSITY OF ARIZONA

UNIVERSITY OF BRITISH COLUMBIA

CALIFORNIA INSTITUTE OF TECHNOLOGY

UNIVERSITY OF CALIFORNIA

UNIVERSITY OF MONTANA

UNIVERSITY OF NEVADA, RENO

NEW MEXICO STATE UNIVERSITY

OREGON STATE UNIVERSITY
UNIVERSITY OF OREGON

UNIVERSITY OF SOUTHERN CALIFORNIA

STANFORD UNIVERSITY

UNIVERSITY OF HAWAII

UNIVERSITY OF UTAH

WASHINGTON STATE UNIVERSITY

UNIVERSITY OF WASHINGTON 


\section{PACIFIC JOURNAL OF MATHEMATICS}

Volume 159 No. $1 \quad$ May 1993

An application of the very weak Bernoulli condition for amenable groups

SCOT ROBERT ADAMS and JEFFREY EDWARD STEIF

An application of homogenization theory to harmonic analysis on solvable Lie groups of polynomial growth

G. Alexopoulos

The standard double soap bubble in $\mathbf{R}^{2}$ uniquely minimizes perimeter

Joel Foisy, Manuel Alfaro Garcia, JefFrey FARlowe

Brock, NiCKELOUS Hodges and JASON ZimbA

Pseudo regular elements and the auxiliary multiplication they induce

BARRY E. JOHNSON

A converse to a theorem of Komlós for convex subsets of $L_{1}$

CHRISTOPHER JOHN LENNARD

General Kac-Moody algebras and the Kazhdan-Lusztig conjecture

WAYNE L. NEIDHARDT

The flow space of a directed $G$-graph

WILLIAM LINDALL PASCHKE

Primitive ideals and derivations on noncommutative Banach algebras

MARK PHILLIP THOMAS

Equivariant Nielsen numbers

PETER N-S WONG

Volumes of tubular neighbourhoods of real algebraic varieties

RichaRd ALEXANDER WONGKEW

The intrinsic group of Majid's bicrossproduct Kac algebra 